\title{
Reply to the Letter to the Editor: Protection of computed tomography suites from SARS-CoV-2 infection in a tertiary emergency hospital
}

\author{
Daisuke Utsunomiya ${ }^{1} \cdot$ Tsuneo Yamashiro $^{1}$
}

Received: 11 April 2020 / Accepted: 13 April 2020 / Published online: 24 April 2020

○ Japan Radiological Society 2020

We appreciate the interest of Dr. Katsumori and Dr. Yoshikawa in our report [1], and we respect their valuable suggestions. First, we agree with the opinion that computed tomography $(\mathrm{CT})$ is not the first line test to diagnose coronavirus disease 2019 (COVID-19). Real-time reverse transcriptasepolymerase chain reaction (RT-PCR) is the reference standard for definitive COVID-19 diagnosis. The infection control unit in the $\mathrm{CT}$ suites requires specialized knowledge, equipment, and manpower. Inadequate preparation and infection protocol may cause in-hospital transmission from the CT suites. Many patients undergoing CT scanning have underlying diseases (e.g., hypertension, diabetes mellitus, malignancy, and so on). The COVID-19 pneumonia is likely to be serious in such patients [2] and, therefore, we have to be careful with infection control in the CT suites. However, CT may play a complementary or alternative role to the standard RT-PCR test because its sensitivity to detect COVID-19 lung diseases is reportedly higher than that of RT-PCR under circumstances of extremely high prevalence rates [3].

They also pointed out another important problem of aerosol infection. Regarding this problem, we addressed this concern in the reply to the letter to the editor from Dr. Joob and Dr. Wiwanitkit.

We examined nine patients (14 examinations) with COVID-19 in our hospital using CT, and performed our infection protection protocol for each of them. There has not been a single in-hospital transmission in the CT suite; however, the number of sampled patients is small.

As suggested, infection protection protocol is also important in magnetic resonance imaging, angiography, nuclear medicine, and radiation therapy suites. We strongly believe that the infection control protocol should be similar in all the radiological suites. Two radiologic technologists should handle the patient, i.e., a "clean" technologist for scanning and a "contact" technologist for setting. Careful cleaning is also necessary after scanning of each COVID-19 patient, and air change should be performed, if necessary.

\section{References}

1. Nakajima K, Kato H, Yamashiro T, Izumi T, Takeuchi I, Nakajima H, et al. COVID-19 pneumonia: infection control protocol inside computed tomography suites. Jpn J Radiol. 2020. https:// doi.org/10.1007/s11604-020-00948-y.

2. Huang C, Wang Y, Li X, Ren L, Zhao J, Hu Y, et al. Clinical features of patients infected with 2019 novel coronavirus in Wuhan, China. Lancet. 2020;395:497-506.

3. Fang Y, Zhang H, Xie J, Lin M, Ying L, Pang P, et al. Sensitivity of chest CT for COVID-19: comparison to RT-PCR. Radiology. 2020. https://doi.org/10.1148/radiol.2020200432.

Publisher's Note Springer Nature remains neutral with regard to jurisdictional claims in published maps and institutional affiliations.

Daisuke Utsunomiya

d.utsunomiya@gmail.com

1 Diagnostic Radiology, Yokohama City University,

3-9, Fukuura, Kanazawa-ku, Yokohama-shi,

Kanagawa 236-0004, Japan 